\title{
Improved Micro Thermal Shear-Stress Sensor
}

\author{
Jin-Biao Huang, Steve Tung, Chih-Ming Ho, Chang Liu, and Yu-Chong Tai
}

\begin{abstract}
Micro hot-film shear-stress sensors have been designed and fabricated by surface micromachining technology which is compatible with IC technology. A polysilicon strip, $2 \mu \mathrm{m} \times 80 \mu \mathrm{m}$, is deposited on top of a thin silicon nitride film and functions as the sensor element. By using the sacrificial-layer technique, a cavity (a vacuum chamber of about 300 mtorr), $200 \times 200 \times 2 \mu \mathrm{m}$, is placed between the silicon nitride film and the silicon substrate. This cavity significantly increases the sensitivity of the sensor by reducing the heat loss to the substrate. The frequency response of the sensor, however, is degraded by the cavity. For comparison purposes, a sensor structure without a cavity has also been designed and fabricated on the same chip. When operated in a constant temperature mode, the cutoff frequencies of the sensors with and without a cavity can reach 9 and $130 \mathrm{kHz}$, respectively. Wind tunnel calibration of the sensor with a cavity shows a sensitivity of about $10 \mathrm{mV} / \mathrm{Pa}$, which is about two orders of magnitude higher than other micromachined shear stress sensors.
\end{abstract}

\section{INTRODUCTION}

$\mathbf{F}$ LUID flow over a solid wall generates a velocity gradient that produces shear stress at the wall. This wall shear stress is one of the important flow properties in aerospace engineering and fluid mechanics [1]. Presently, there are many measurement techniques for shear stress. However, none of them can simultaneously satisfy the operational requirements for turbulent boundary layer research: fine spatial resolution $(\sim 100 \mu \mathrm{m})$, fast frequency response $(>5 \mathrm{kHz})$, and high sensitivity. With the help of micromachining technology, the floating-element shear stress sensor has satisfied the requirement of spatial resolution [2]-[3]. But the other requirements remain unresolved.

An new micromachined shear-stress sensor based on hotfilm anemometry has been proposed and designed in the present study [4] for a distributed microelectromechanical system (MEMS), which can achieve all of the above requirements. A hot-film sensor is an example of indirect shear-stress measurement. The heat transfer from a resistively heated element to the flow is measured, and from this, a value for shear stress is inferred. Although this type of probe has been employed widely, the factors influencing its unsteady performance are not yet well understood [1], [5]. It is well accepted that the thermal properties of the substrate affect sensor operation [5]-[6]. Some workers have attempted to

Manuscript received April 24, 1995; revised January 11, 1996. This work was supported by the AFOSR URI Project (University Research Institute).

J.-B. Huang was with the Center for Micro Systems, University of California, Los Angeles, CA 90095-1597 USA. He is now with the School of Electrical Engineering, Cornell University, Ithaca, NY 14853 USA.

S. Tung and C.-M. Ho are with the Center for Micro Systems, University of California, Los Angeles, CA 90095-1597 USA.

C. Liu and Y.-C. Tai are with the Department of Electrical Engineering, California Institute of Technology, Pasadena, CA 91125 USA.

Publisher Item Identifier S 0018-9456(96)03495-X.

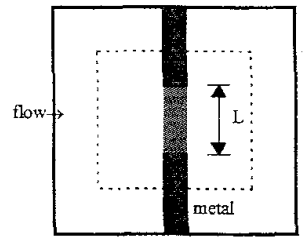

(a)

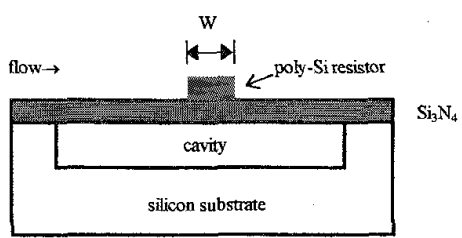

(b)
Fig. 1. (a) Schematic top, and (b) cross-sectional views of the micro hot-film shear-stress sensor.

isolate the sensor from the substrate [7]-[8], but they were not able to achieve the desired high-frequency response. The effect of insulation on the sensor's performance remains mostly unknown. Although some experimental studies and simulations have been carried out [5]-[6] on the conventional hot-film probes, to the authors' knowledge, none has been carried on the micromachine-based sensors which provide a better understanding of the probes' performance.

In this paper, theoretical and experimental studies of the importance of insulation on the sensor's performance are discussed. Two kinds of hot-film shear-stress sensors, one with a cavity (vacuum chamber) underneath to insulate it from the substrate and the other without a cavity, have been designed and fabricated by micromachining technology. Except for the vertical structure, the two sensors have identical geometry. A description of the sensor structure and process can be found in Section II. The theoretical models and analytical solutions for the two sensors will be introduced in Section III. The experimental results concerning the spatial resolution, frequency response, and sensitivity of the sensors are then described in Section IV. Finally, the conclusions are given in Section V.

\section{SENSOR AND STRUCTURE}

Fig. 1 schematically shows the top and cross-sectional views of the micro hot-film sensor with a cavity underneath. By using micromachining technology, a vacuum chamber (cavity), the square part (dashed line) in Fig. 1(a), is placed under a thin silicon nitride film. The size of the cavity is $200 \times 200 \times 2 \mu \mathrm{m}$. A polysilicon strip, $2 \times 80 \mu \mathrm{m}$, is deposited on the silicon nitride film and functions as the sensor element. Metal leads connect the sensor element to the outside through bonding pads. A similar sensor without a cavity underneath is also included on the chip. Both sensors, with and without a cavity, have identical geometry. The flow direction is indicated in Fig. 1.

The cavity is formed by a sacrificial-layer (PSG, phosphosilicate glass) technique. Both silicon nitride and polysilicon layers are deposited by LPCVD (low-pressure chemical vapor deposition). The aluminum metallization forms the 


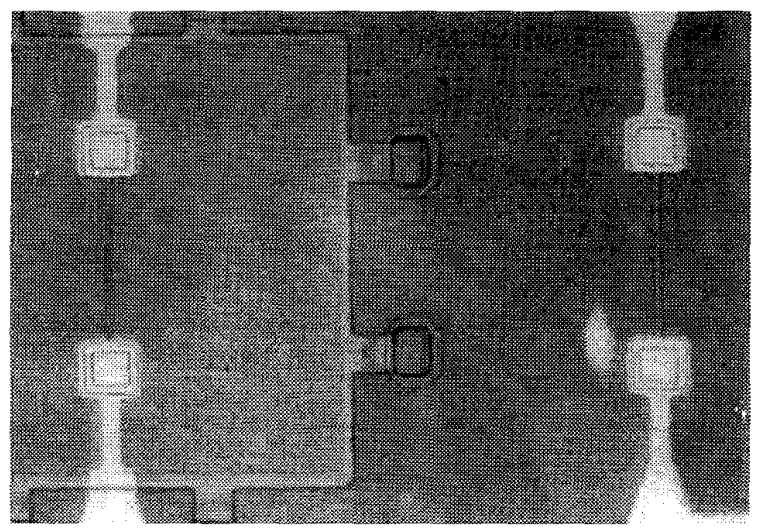

Fig. 2. Photograph of the microfabricated hot-film shear-stress sensors with a cavity (left) and without a cavity (right) underneath. Both sensors have same element size of $2 \times 80 \mu \mathrm{m}$.

metal leads. The polysilicon resistor is uniformly doped by phosphorus to a low sheet resistance of typically $50 \Omega / \square$. The measured TCR (temperature coefficient of resistivity) of the sensor at this doping level is about $0.09 \% /{ }^{\circ} \mathrm{C}$. The detailed process steps are given in [4].

A photograph of the microfabricated sensors is shown in Fig. 2. The one on the left is the sensor with a cavity, and the one on the right is the sensor without a cavity. Due to optical reflection, the metal leads appear in white. The cavity is not shown thoroughly, but its frame can still be observed. The theoretical models for these sensors are given in the following section.

\section{THEORETICAL MODELS}

A general three-layer structure is used to characterize the dynamic performance of the micro sensors, as shown in Fig. 3(a). The film layer at the top represents the sensor element. The insulation layer represents the silicon nitride diaphragm. The silicon substrate with high thermal conductivity is treated as a heat sink. The length and width of the sensors are $L$ and $W$ (as shown in Fig. 1), respectively. The $q$ in the figure represents heat transfer. The subscripts $f, i$, and $c$ represent the heat transfer to the film, the insulation layer, and the convective heat transfer to the measured fluid, respectively. The same subscripts are used for the layer thickness $d$ and other thermal parameters. For the case of the sensor with a cavity underneath, heat $q_{i}$ can be considered to go laterally first and then vertically to the heat sink (substrate), as shown in Fig. 3(b).

The energy balance equation in the general structure of Fig. 3(a) can be described as follows:

$$
i^{2} R=c_{f} m_{f} \frac{d T_{f}}{d t}+c_{i} m_{i} \frac{d T_{i}}{d t}+h\left(u_{\tau}\right) A\left(T-T_{o}\right) .
$$

The heating current $i$ through the resistive sensor $R$ produces heating power $i^{2} R$. The power is dissipated or stored in the three ways which correspond to the three terms on the righthand side of the above equation:

1) Convective heat transfer $q_{c}=h\left(u_{\tau}\right) A\left(T-T_{o}\right)$ to the sensor's environment, in which $T$ and $T_{o}$ are the temperatures of the sensor element and of the heat sink,

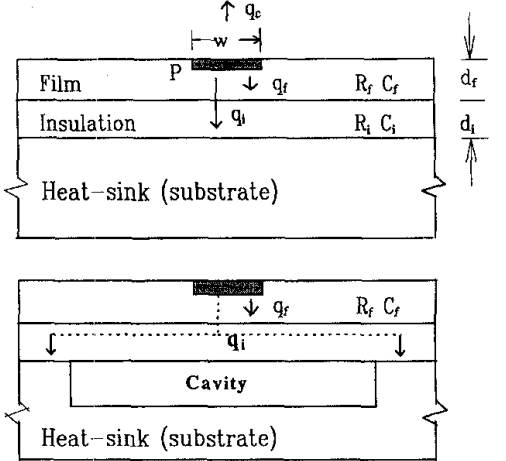

(a)

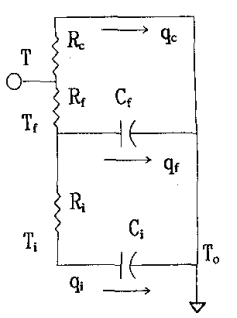

(b)
Fig. 3. Heat transfer models for the general sensor with (a) an insulation layer underneath, (b) the sensor with a cavity underneath, and (c) their electric-analogy's equivalent circuit.

respectively. The convective heat transfer coefficient is represented by $h\left(u_{\tau}\right)$ and it is a function of shear velocity $u_{\tau}$. The relationship between the shear velocity $u_{\tau}$ and the wall shear stress $\tau_{w}$ is $\tau_{w}=u_{\tau}^{2} \rho$ where $\rho$ is the measured fluid density. The heat transfer to the insulation layer, substrate, and then the measured environment is also included in this term. This makes the effective heat transfer area a little larger than $W \times L$.

2) Conductive heat transfer $q_{f}$ to the film. This is equal to the energy stored in the film, $q_{f}=c_{f} m_{f} d T_{f} / d t$, where $c_{f}, m_{f}$, and $T_{f}$ are the specific heat, mass, and temperature of the film layer, respectively.

3) Conductive heat transfer to the insulation layer and stored in it, $q_{i}=c_{i} m_{i} d T_{i} / d t$, which has the same parameter notation for the insulation layer as in the film case.

The last two heat transfer terms are related to the thermal conduction in the following way:

$$
\begin{gathered}
q_{i}=c_{i} m_{i} \frac{d T_{i}}{d t}=\frac{k_{i} A\left(T_{f}-T_{i}\right)}{d_{i}} \\
q_{f}+q_{i}=\frac{k_{f} A\left(T-T_{f}\right)}{d_{f}}
\end{gathered}
$$

where $k_{i}$ and $k_{f}$ are the thermal conductivity of insulation and film layers, respectively.

Considering the fluctuating time-dependent part of the variables above, neglecting secondary terms, and applying Laplace transforms, the transfer function between the sensor's temperature change $\Delta T$ and the input variable $\Delta F$ can be obtained as follows:

$$
\begin{gathered}
\frac{\Delta T}{\Delta F}=\left(\frac{\alpha_{R}}{\alpha i^{2} R_{o}}\right) \frac{\tau_{2} s+1}{\tau_{1} \tau_{2} s^{2}+\left(\tau_{1}+\tau_{2}+\tau_{3}\right) s+1} \\
\Delta F=\frac{\partial P}{\partial i} \Delta I-\left(\bar{T}-T_{o}\right) A \Delta H
\end{gathered}
$$

where $\Delta F$ is the Laplace transform form of the input variable, which is a function of both the electrical current input perturbation $\Delta I$ and the shear-stress related input perturbation 
$\Delta H . \bar{T}$ is the static temperature of the sensor. $\alpha_{R}, \alpha$, and $R_{o}$ in (4) are the resistive overheat ratio (defined as $\left(R-R_{0}\right) / R_{0}$ in which $R$ is the sensor resistance at the temperature $T$ ), the temperature coefficient of resistivity $(\mathrm{TCR})\left(1 /{ }^{\circ} \mathrm{C}\right)$ of the sensor element, and the sensor resistance at temperature $\mathcal{T}_{o}$, respectively. The time contents $t_{1}, t_{2}$, and $t_{3}$ in (4) are

$$
\begin{aligned}
\tau_{1} & =\frac{\alpha_{R}}{\alpha i^{2} R_{o}} c_{f} m_{f} \\
\tau_{2} & =\frac{d_{i}}{k_{i} A} c_{i} m_{i} \\
\tau_{3} & =\frac{\alpha_{R}}{\alpha i^{2} R_{o}} c_{i} m_{i} .
\end{aligned}
$$

Although the system described by (4) is second order, it can be accepted that the pole $s_{1} \approx-1 /\left(\tau_{1}+\tau_{2}+\tau_{3}\right)$ is dominant, and hence can be approximated to a first-order system with a time constant of

$$
\tau_{c}=\tau_{1}+\tau_{2}+\tau_{3}
$$

This total time constant consists of three parts: the first part $\tau_{1}$ is exactly the same as the time constant found by previous workers [9]; the last two parts, $\tau_{2}$ and $\tau_{3}$, obtained here indicate how the insulation layer parameters affect the sensors' dynamic performance. From (6) and (7), it can be seen that the existence of the insulation layer tends to increase the time constant and decrease the frequency response of the system. The smaller the thermal conductivity $k_{i}$ of the insulation layer, the larger the effect. The larger the specific heat $c_{i}$ and mass $m_{i}$ of the insulation layer, the longer the time constant. This also explains why high dynamic performance cannot be achieved by insulating the sensor from the substrate.

For the sensor with a cavity underneath, heat goes laterally first in the insulation layer and then vertically to the heat sink. This changes the time constant $\tau_{2}$ to

$$
\tau_{2}=\frac{L_{c}}{2 k_{i} d_{i} L} c_{i} m_{i}
$$

where $L_{c}$ is the half length of the cavity side and the " 2 " in the denominator is a result of the bidirectional heat transfer in the insulation layer. Both $\tau_{1}$ and $\tau_{3}$ remain the same. Since $L_{c}$ in (8) is much larger than $d_{i}$ in (6) while $2 d_{i} L$ in (8) and $A$ in (6) are of the same order, the time constant $\tau_{2}$ of the sensor with a cavity underneath is longer than that without a cavity. Actually, the effective heat transfer area $A$ and effective masses $m_{f}$ and $m_{i}$ also increase because a larger volume is heated up due to the existence of the cavity. All of these factors tend to make the total time constant $\tau$ of the sensor with a cavity longer than that without a cavity.

It should be mentioned that an Si substrate is not an ideal heat sink. Part of the substrate behaves just like an insulation layer. This makes the actual time constant a little bit longer than that indicated by (6)-(8).

The sensitivity of the system can also be obtained based on (4) and (5) by putting $\Delta F=1 / s$ into (4):

$$
\Delta T=\frac{\alpha_{R}}{\alpha i^{2} R_{o}} \frac{\left(\tau_{2} s+1\right)(1 / s)}{\tau_{1} \tau_{2} s^{2}+\left(\tau_{1}+\tau_{2}+\tau_{3}\right) s+1} .
$$

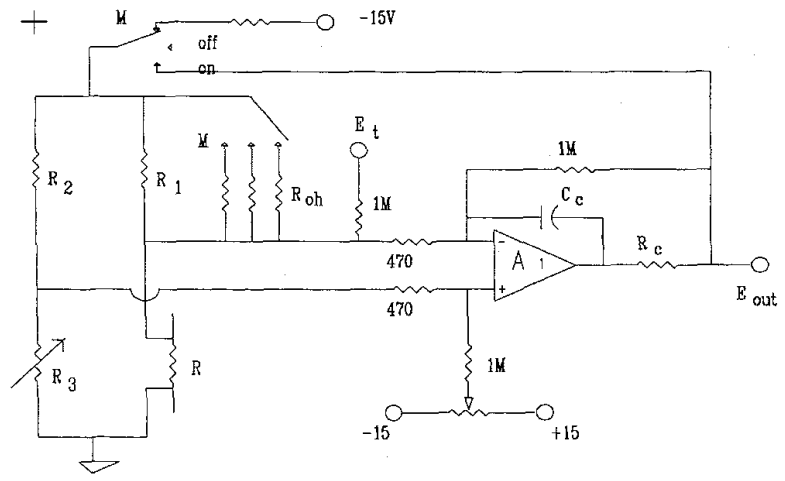

Fig. 4. The constant-temperature circuit for the hot-film shear-stress sensor in which $R$ is the sensor and $E_{t}$ is the terminal for the testing signal.

This equation can be rearranged into the following form:

$$
\Delta T=\frac{K_{0}}{s}+\frac{K_{1}}{\left(s-s_{1}\right)}+\frac{K_{2}}{\left(s-s_{2}\right)}
$$

where $s_{1}$ and $s_{2}$ are the two poles of the system and $K_{0}$, $K_{1}$, and $K_{2}$ are constants. From (9) and (10), we can get the sensitivity coefficient $K_{0}$ :

$$
K_{0}=\left.s \Delta T\right|_{s=0}=\frac{\alpha_{R}}{\alpha i^{2} R_{o}} .
$$

By comparing (6) and (11), it can be seen that the sensitivity has a conflicting requirement with the system's time constant.

Alternatively, the electric analogy of the heat transfer can be used, and the equivalent circuit is shown in Fig. 3(c). The quantity $d / A k$ in (2) and (3) is equivalent to a thermal resistance $R_{i}$ for the insulation layer or $R_{f}$ for the film layer. Similarly, the quantity $\mathrm{cm}$ in (1) is equivalent to a thermal capacitance $C_{i}$ or $C_{f}$. The same result but with a clearer physical meaning can be obtained by using the electric analogy method, e.g., $\tau_{2}=R_{i} C_{i}$.

\section{EXPERIMENTS}

After the fabrication of the hot-film sensor, both constant current (CC) and constant-temperature (CT) circuits have been designed for the sensor operation. In this paper, we focus on the CT mode, as illustrated in Fig. 4. In the figure, $R$ is the sensor, $R_{o h}$ is for adjusting the resistive overheat ratio, and $R_{c}$ and $C_{c}$ are for compensation. Since suitable velocity fluctuations are not readily available, electronic test signals are usually used to determined the time constant of the system. According to both theoretical analyses [10] and experimental confirmation [2], the frequency response or time constant can be obtained by feeding an electronic sine wave or square wave into the CT circuit. The terminal $E_{t}$ in Fig. 4 is for this purpose.

The square-wave responses of both sensors with and without a cavity are measured and shown in Fig. 5(a) and (b), respectively. Based on the established method for traditional hot-film shear stress sensors [5], [7]-[10], the time constants 


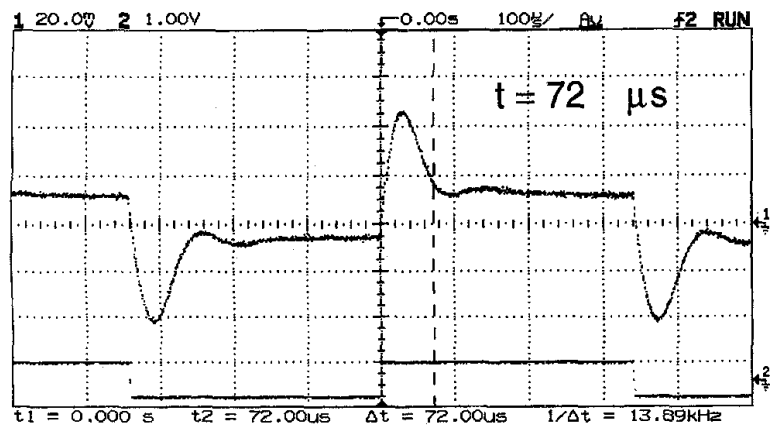

(a)

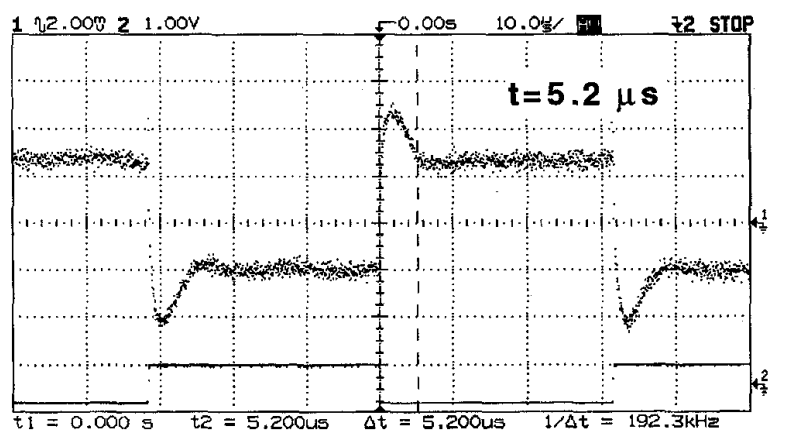

(b)

Fig. 5. Square wave responses of the hot-film sensors (a) with, and (b) without a cavity underneath. The time constants are 72 and $5.2 \mu$ s for the top one and the bottom one, respectively.

of the two sensors can be measured. The measured time constants are 72 and $5.2 \mu$ s for the former and the latter, respectively. The sensor without a cavity clearly has a much shorter (about one order of magnitude) time constant. This is consistent with the theoretical results presented in Section III. An overheat ratio of 0.12 is used in this experiment. Based on the approximate relationship between the time constant $\tau_{c}$ and the cutoff frequency $f_{c}$ for CT operation, $f_{c}=1 /\left(1.5 \tau_{c}\right)$, the cutoff frequencies of the sensors can be estimated. They are 9 and $128 \mathrm{kHz}$ for the sensors with a cavity and without a cavity, respectively.

The frequency responses have been obtained by using an electronic sine wave instead of a square wave. Fig. 6 presents the experimental results for both sensors. The cutoff frequencies are 9 and $130 \mathrm{kHz}$, respectively. This is consistent with the estimates based on time constant measurements.

It should be mentioned that the experiments are carried out in a CT mode with a feedback. That is why the step response results shown in Fig. 5 are second order.

Calibration of both sensors is carried out in the fully turbulent region of a wind tunnel. The shear-stress values are obtained from streamwise pressure gradient measurements, $\tau_{w}=-0.5 h(d p / d x)$, where $h$ is the height of the wind tunnel. The calibration is carried out at constant temperature mode and an overheat ratio of 0.12 . The calibration result is shown in Fig. 7. It can be seen that the sensitivity of the sensor with a cavity is about $10 \mathrm{mV} / \mathrm{Pa}$, which is about one order of magnitude higher than that without a cavity and about

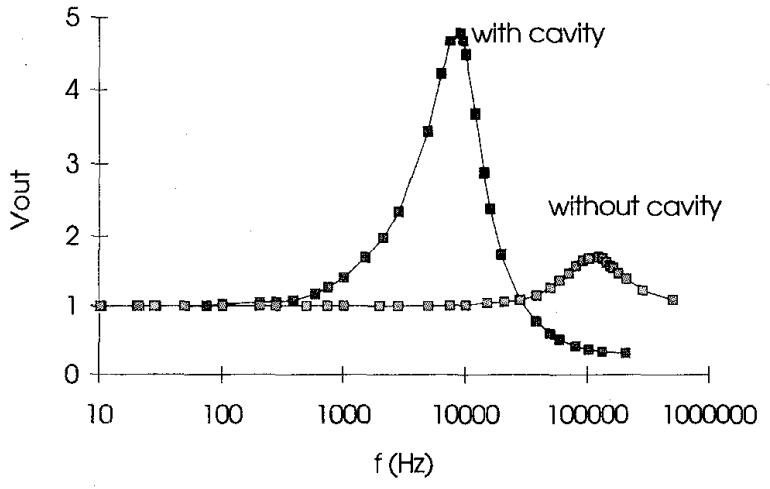

Fig. 6. Frequency responses of the micro hot-film sensors with and without cavity. The cutoff frequencies are 9 and $130 \mathrm{kHz}$ for the former and the latter, respectively.

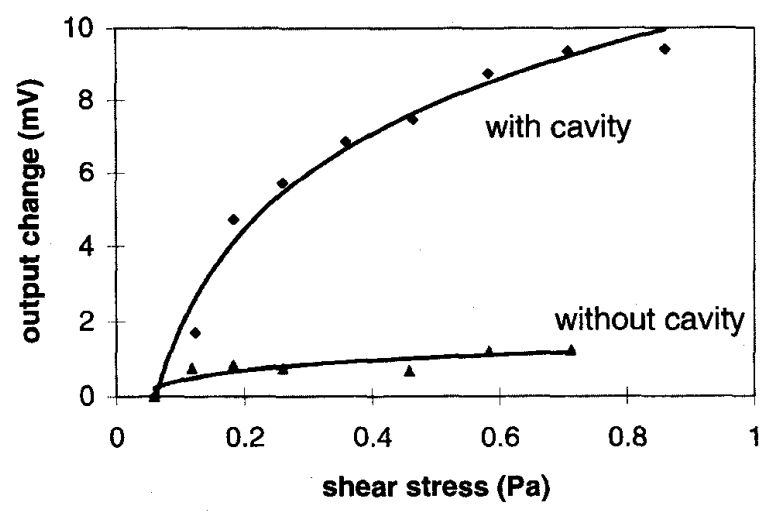

Fig. 7. Calibration result of voltage output change versus shear stress at constant temperature operation mode (the resistive overheat ratio is $12 \%$ ).

two orders of magnitude higher than other micromachined shear-stress sensors.

\section{CONCLUSIONS}

The micromachined hot-film shear-stress sensors with and without a cavity underneath have been designed and fabricated by micromachining technology. Theoretical heat transfer models and analytical solutions indicate that the insulation layer has an important effect on the sensors' frequency response and sensitivity. Both theoretical and experimental results show that the sensor with a cavity underneath has a slower frequency response and a higher sensitivity than the sensor without a cavity. There is a conflict in the requirement between frequency response and sensitivity. The typical element size, cutoff frequency, and sensitivity of the sensor with a cavity are $2 \times 80 \mu \mathrm{m}, 9 \mathrm{kHz}$, and $10 \mathrm{mV} / \mathrm{Pa}$.

\section{REFERENCES}

[1] T. J. Hanratty and J. A. Cambell, "Measurement of wall shear stress," in Fluid Mechanics Measurements, R. J. Goldstein, Ed. New York: Hemisphere, 1983, pp. 599-615.

[2] J. Shajii, K.-Y. Ng, and M. A. Schmidt, "A microfabricated floating-element shear stress sensor using wafer-bonding technology," IEEE/ASME J. Microelectromech. Syst., vol. 1, no. 2, pp. 89-94, 1992.

[3] M. A. Schmidt, R. T. Howe, S. D. Senturia, and J. H. Haritonidis, "Design and calibration of a microfabricated floating-element shear- 
stress sensor," IEEE Trans. Electron Devices, vol. 35, pp. 750-757, June 1988.

[4] C. Liu, Y.-C Tai, J-B. Huang, and C.-M. Ho, "Surface micromachined thermal shear stress sensor," in ASME Symp. Application of Microfabrication to Fluid Mech., Chicago, IL, 1994, pp. 9-11.

[5] M. J. Moen and S. P. Schneider, "The effect of sensor size and substrate properties on the performance of flush-mounted hot-film sensors," in Thermal Anemometry - 1993, D. E. Stock, S. A. Sherif, A. J. Smits, and J. Davidson, Eds. New York: ASME, 1993, pp. 249-261.

[6] S. Albin, A. Bulusu, S. D. Martinson, and D. S. Gray, "Frequency response simulations of a diamond based sensor for supersonic flows," in Thermal Anemometry-1993, D. E. Stock, S. A. Sherif, A. J. Smits, and J. Davidson, Eds. New York: ASME, 1993, pp. 181-184.

[7] C. O. Ajagu and P. A. Libby, "Modified gauge for time-resolved skin-friction measurements," Rev. Sci. Instrum., vol. 53, no. 12, pp. 1920-1926, 1982

[8] D. Reda, "Rise-time response of nickel-foil-on-Kapton-substrate, hotfilm shear stress sensors," AIAA Paper 91-0169, 1991.

[9] R. F. Blackwelder, "Hot-wire and hot-film anemometers," in Methods of Experimental Physics: Fluid Dynamics, R. J. Emrich, Ed. New York: Academic, 1981, pp. 259-314.

[10] P. Freymuth, "Frequency response and electronic testing for constanttemperature hot-wire anemometers," J. Phys. E: Sci. Instruments, vol. 10, pp. 705-710, 1977 .

Jin-Biao Huang received the B.Sc., M.Sc., and Ph.D. degrees in electrical engineering from Nanjing Institute of Technology (now Southeast University), Nanjing, China, in 1983,1986, and 1989, respectively.

After completing his education. he joined the Microelectronics Center Southeast University, where he was an Associate Professor from 1992 to 1993. In 1993, he became a Research Associate in the Center for Micro Systems, University of California, Los Angeles, working in the area of sensors, MEMS, electronic measurements, and fluid mechanics. He is currently a Visiting Scientist at Cornell Nanofabrication Facility (formerly NNF) and working for Boonton Electronics Corporation through the New Jersey Institute of Technology, Newark. His research interests include integrated sensors, temperature sensors, vacuum sensors, shear-stress sensors, actuators, MOS devices, CMOS IC's design, and electronic measurements.

Steve Tung received the B.S. degree in mechanical engineering from the National Taiwan University in 1984 , and the Ph.D. degree from the University of Houston, Houston, TX, in 1992.

After receiving the Ph.D. degree, he became a Lecturer in the Department of Mechanical Engineering, University of Houston. In 1993, he joined the Center for Micro Systems (CMS), University of California, Los Angeles, as a Research Associate, working in the area of active drag reduction through the implementation of MEMS technology. His research interests include turbulence control, micromachine-based sensors and actuators, aerodynamics, aeroacoustics, and multiphase flows.

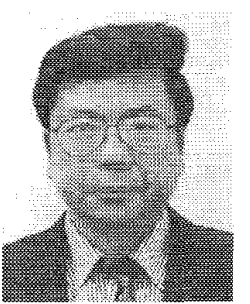

Chih-Ming Ho received the B.S. degree in mechanical engineering from National Taiwan University in 1967, and the Ph.D. degree in mechanics from The Johns Hopkins University, Baltimore, MD, in 1974.

$\mathrm{He}$ is currently a Professor in the Department of Mechanical, Aerospace and Nuclear Engineering Department, University of California, Los Angeles, and is the Director of the Center for Micro Systems (CMS).

Dr. Ho was elected Fellow of the American Physical Society for pioneering research in controling the evolution of turbulent free shear layers. He was elected Fellow of the American Institute of Aeronautics and Astronautics for his seminal contributions to the basic understanding and control of turbulent shear flows and for pioneering contributions to applying microtransducers to aerospace science. He has published more than 100 papers in the areas of turbulence, MEMS, aerodynamics, aeroacoustics, and biofluid dynamics. He was an Associate Editor of the ASME Journal of Fluids Engineering from 1990 to 1993 and was an Associate Editor of the AIAA Journal from 1985 to 1987.

Chang Liu received the B.S. degree in precision instrumentation from Tsinghua University, Beijing, in 1990, and the M.S. degree in electrical engineering from the California Institute of Technology, Pasadena, in 1991.

He is currently working toward the Ph.D. degree in micromachining and microelectro mechanical systems, under Prof. Yu-Chong Tai of Caltech. He has been working on several micromachining projects, including an integrated silicon atomic force microscope probe, a pressure sensor, a hotwire anemometer and a surface shear-stress sensor, two different types of magnetic actuators, and a MEMS for fluid control.

Yu-Chong Tai received the B.S. degree in electrical engineering from the National Taiwan University in 1981, and the M.S. and Ph.D. degrees from the University of California, Berkeley, in 1986 and 1989, respectively.

After completing his education, he joined the Faculty of Electrical Engineering at the California Institute of Technology, Pasadena, where he is currently an Assistant Professor. His research interests have included silicon micromachining and its applications, such as neural prosthetic devices, integrated millimeter wave waveguides, micro fluidic devices, miniature harddisk drives, microphones, silicon hot-wire anemometers, magnetic milli- and microactuators, shock sensors, and MEMS systems for active fluid control.

Dr. Tai is a member of the Editorial Board of the Journal of Micromechanics and Microengineering. He received the Presidential Young Investigator Award and the David and Lucile Packard Fellowship in 1991. 